\title{
A Systematic Review of Coagulase-Negative Staphylococci in Neonatal Sepsis
}

\author{
John-Ugwuanya A Grace ${ }^{1 *}$ and Stephen K Obaro ${ }^{2}$ \\ ${ }^{1}$ International Foundation Against Infectious Disease in Nigeria, Abuja, Nigeria \\ ${ }^{2}$ Division of Pediatric Infectious Disease University of Nebraska Medical Center, Omaha, Nebraska, Nigeria
}

"Corresponding author: John-Ugwuanya A Grace, Department of Pharmaceutics and Pharmaceutical Microbiology, Ahmadu Bello University, Zaria, Kaduna, Nigeria, Tel: +2347061145614; E-mail: graceamy2k@yahoo.com

Received date: January 18, 2019; Accepted date: February 06, 2019; Published date: February 12, 2019

Copyright: $\odot 2019$ Grace JA, et al. This is an open-access article distributed under the terms of the Creative Commons Attribution License, which permits unrestricted use, distribution, and reproduction in any medium, provided the original author and source are credited.

\begin{abstract}
We sought to analyze the prevalence of neonatal sepsis based on eight selected articles published in 2016 and 2017 with details on the demographics, bacteria distribution, risk factors, antibacterial susceptibility and the rising isolation of Coagulase-negative staphylococci (CoNS) among other bacteria isolates. Early Onset Neonatal Sepsis (EONS) was prevalent in most of the studies than Late-Onset Neonatal Sepsis (LONS). The rate of CoNS isolation in neonatal blood cultures was significantly high with varying mortality, morbidity and methicillin-resistant CoNS observed. However, CoNS is still considered as a contaminant when the clinical course is not consistent with sepsis. The predominant species were Staphylococcus epidermidis, S. haemolyticus, S. hominis and S. capitis, which were being regarded as conditional pathogens or contaminants. Staphylococcus capitis NRCS-A clone was identified in 17 countries with marked multidrug resistance. High susceptibility of CoNS to linezolid and vancomycin were observed, except in S. capitis NRCS-A clone that showed treatment failure to aminoglycosides and vancomycin. Research on CoNS in neonatal sepsis will be a thriving area for years to come especially with its close association with hospital routine, assessing its pathogenic potential, the global spread of the multidrug-resistant NRCS-A clone of $S$. capitis and the introduction of vaccination opportunities. Adequate funding and collaboration of research effort will be required to address these key questions.
\end{abstract}

Keywords: Staphylococcus aureus, Coagulase-negative staphylococci; Neonatal sepsis

\section{Introduction}

Neonatal sepsis is a systemic inflammatory response caused by bacterial production of toxins after gaining entry into the bloodstream and multiplying [1]. It is classified into the early-onset neonatal sepsis ( $\leq 72 \mathrm{hrs}$ ) due to bacteria acquired before or during delivery, and lateonset neonatal sepsis ( $>72$ hrs-28days) involving pathogens acquired after delivery [2,3]. Generally, it is a life-threatening case. In preterm babies, this can cross the blood-brain barrier since the baby is not fully developed, which might result in increased morbidity and mortality globally $[1,4]$. This demands immediate empirical antibiotic treatment after collection of blood culture samples [5].

In sub-Saharan Africa, neonatal mortality is high with one-third of the death resulting from septicemia [6]. However, there is minimal data on antibiotic resistance of frequently isolated invasive pathogens and their clinical application in neonatal sepsis. The pathogens include Gram-positive cocci, Gram-negative bacteria and some fungi [1]. This varies based on geographical location, antibiotic usage, time of onset of illness and the use of intravascular medical devices. Globally, about $40 \%$ of deaths in children under five from developed and developing countries occurs in the neonatal period ( $\leq 28$ days) with predominance in developing and low-income countries [2]. Empirical treatment according to the World Health Organization is recommended for neonates having sepsis in areas of sub-Saharan Africa that lacks wellequipped microbiology laboratory [6].
The isolation of Gram-positive cocci in neonatal sepsis has increased significantly in the last two decades, especially with the use of invasive medical devices and the onset of resistant organisms [3]. Coagulase-negative staphylococci have been identified as the common Gram-positive cocci in neonatal sepsis, especially in low birth weight infants [4,7]. It is often associated with a prolonged hospital stay, indwelling medical devices and their ability to colonize and form biofilm [8]. Ascertaining their clinical significance is difficult since they are skin commensals and could contaminate blood culture samples [6]. Different species of clinical significance include $S$. epidermidis, $S$. haemolyticus, $S$. hominis and $S$. capitis [1,7]. Klebsiella species is the main Gram-negative bacteria in several developing countries [2].

Clinical manifestations of neonatal sepsis are non-specific. However, suggestive features include hypothermia, fever, failure to thrive, tachycardia, bulging anterior fontanelle, irritability and bacterial isolation from a bloodstream infection. This makes diagnosis and management of the neonatal sepsis difficult. Neonates are susceptible to sepsis due to a poorly developed immune system $[2,4,9]$. The common neonatal risk factors are low fetal birth weight, gestational age, prematurity, fetal distress and perinatal asphyxia. Prolonged labor, vaginal bleeding, urinary tract infection, premature rupture of membrane and lower segment cesarean section are the maternal risk factors [2]. These are closely associated with the mortality rate. Others include child health-care facilities, maternal nutrition, HIV infection, hygiene condition during labor and perinatal care $[3,8]$. Preterm infants having neonatal sepsis are at risk of developing complications due to poorly developed immunity. 


\section{Methods}

A systematic review of eight randomly selected journals on neonatal sepsis published between 2016 and 2017 was carried out. The focus was on the demographics, prevalence, risk factors for infection, antibiotic susceptibility and treatment plan of Coagulase-negative staphylococci in neonatal sepsis. The geographical location of the publications was India, China, France, Nigeria, Zambia, Kenya, and Sweden. Neonates who had already received antibiotics before admission and whose parents declined to give consent were excluded from the study.

\section{Results}

The sex, age, clinical outcome, duration of the study and laboratory diagnosis of the reviewed articles is indicated in Figure 1. Information on the risk factors for neonatal sepsis, bacterial isolate yield, antibiotic susceptibility pattern, mortality rate, and recommended treatment was obtained. A study by Kabwe, et al., [5] of blood cultures with suspected sepsis from 313 neonates of University Teaching Hospital, Lusaka, Zambia between October 2013 and May 2014 had 36\% (113/313) yielding positive blood cultures with 10 disregarded as contaminants, excluding CoNS. Among the 103 culture positive neonatal sepsis, $85 \%$ (88/103) were diagnosed with early-onset neonatal sepsis. Although their initial definition of EONS was 0-7 days; it was subdivided into $\leq 3$ days (43/103) and 4-7 days (45/103) while late-onset neonatal sepsis was $\geq 7$ days (15/103). Using the classification of EONS as $\leq 3$ days in this review, about $69.8 \%$ mortality (30/43) was observed while the rest were discharged. Overall mortality in the negative culture was $31.5 \%$ and $63 \%$ in the positive cultures, which was attributed to an outbreak of Klebsiella species with 94\%-99\% antimicrobial resistance to thirdgeneration cephalosporins. The organisms isolated were Klebsiella species $75 \%$, Coagulase-negative staphylococci $6.8 \%$, Staphylococcus aureus 5.8\%, Escherichia coli $4.9 \%$ and Candida species $4.9 \%$ amongst others (Figure 2).

In a prospective study by Shivanna, et al., [3] carried out from July to August 2014 in a tertiary care hospital in India, among the 50 neonatal blood culture samples obtained, 30\% (15/50) were blood culture positive. It was distributed as Gram-positive cocci 80\% (12/15) and Gram-negative bacilli 20\% (3/15). The predominant bacterial isolate was Coagulase-negative staphylococci as $66.6 \%$ with marked sensitivity to linezolid and vancomycin. CoNS were considered a pathogen with clinical evidence of infection and two positive blood culture yielding the same organism. Methicillin resistance was observed in $60 \%$ of the CoNS isolates. Early onset neonatal sepsis 11 (73.3\%) was predominant than late-onset sepsis 4 (26.7\%).

According to a laboratory-based retrospective investigation conducted in 47 Neonatal Intensive Care Units (NICU) in France, Staphylococcus capitis NRCS-A clone was found in 43 NICUs (91.5\%) and occurring as $46 \%$ of all the positive blood culture [7]. The risk of therapeutic failure was high due to the low susceptibility of NRCS-A isolates to vancomycin and aminoglycosides, which is often used for the treatment of methicillin-resistant Coagulase-negative staphylococci. A broad study of neonatal blood cultures having the NRCS-A clone was also analyzed and found on 154 methicillinresistant S. capitis strains obtained between 1994 and 2015 from seventeen countries: Belgium, Denmark, Finland, Switzerland, Germany, New Zealand, France, Norway, Netherlands, Australia, United Kingdom, Brazil, Canada, United States, Taiwan, South Korea and Czech Republic.
Another Swedish study carried out by Ehlersson, et al., on 332 CoNS isolates from neonatal blood cultures collected within 1987 to 2014 had the CoNS speciated using matrix-assisted laser desorption/ ionization time-of-flight mass spectrometry (MALDI-TOF MS). The susceptibility pattern of $S$. capitis to vancomycin and its clonality to NRCS-A were determined [8]. Although $S$. epidermidis (67.4\%), $S$. haemolyticus $(10.5 \%)$ and $S$. capitis $(9.6 \%)$ were the predominant species, methicillin resistance in $S$. capitis was $75 \%$ with $44 \%$ being multidrug-resistant. Pulsotype NRCS-A was found in $S$. capitis strain CR01.

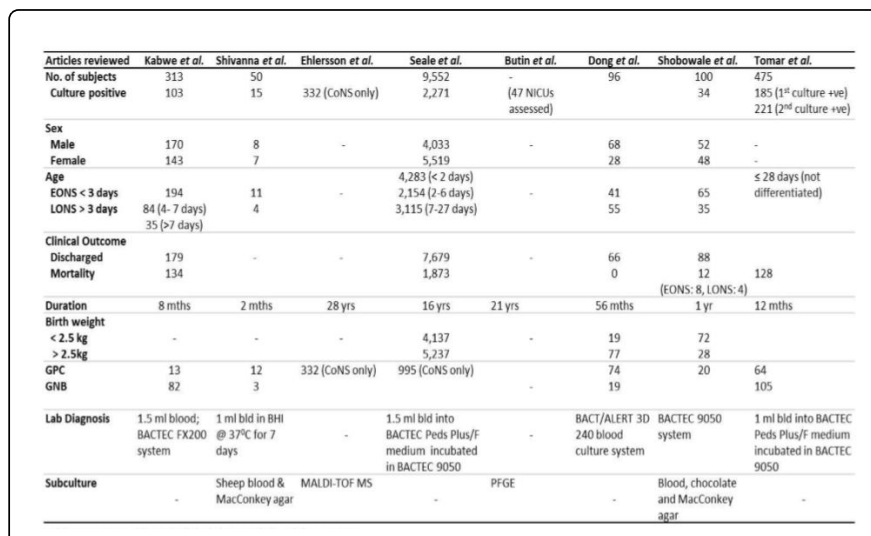

Figure 1: Demographics and Clinical characteristics of the neonates. EONS/LONS: Early/Late-onset neonatal sepsis, MALDI-TOF MS: Matrix-assisted laser desorption/ionization-time of flight mass spectrophotometry, BHI: Brain Heart Infusion broth, NICUs: Neonatal Intensive Care Unit, PFGE: Pulse Field Gel Electrophoresis.

Tomar, et al., designed a prospective cohort study to evaluate the simultaneous collection of two blood cultures within August 2014 to July 2015 from 475 neonates with the rate of pathogen detection compared to the routine practice of single blood culture [9]. There was a $7.6 \%$ increase in isolation rate from taking two blood cultures with $E$. coli $(13.3 \%)$ and $S$. aureus $(11.8 \%)$ being the predominant isolate. However, all the CoNS (1.2\%) were considered as contaminants. Mortality rate was high among neonates that yielded the same organism in the two blood culture samples and had similar sensitivity pattern.

In a retrospective study conducted at Bengbu Third People's Hospital, China from January 2010 to August 2014, a total of 96 cases of neonatal sepsis having positive blood culture results were shown to yield 97 bacteria isolates [1]. This comprised Gram-positive cocci (76.3\%), Gram-negative bacilli (19.6\%) and fungi (4.1\%). Coagulasenegative staphylococci $(70.1 \%)$ were the predominant Gram-positive bacteria with species such as $S$. epidermidis (44.3\%), $S$. haemolyticus $(14.4 \%)$ and $S$. hominis (8.2\%). Klebsiella pneumoniae (10.3\%) and Escherichia coli $(6.2 \%)$ were the common Gram-negative bacteria. Clinical diagnosis was aided by hematological parameters.

Shobowale, et al., carried out a retrospective study of blood cultures at Babcock University Teaching Hospital, Ogun State, Nigeria on 100 neonates meeting the inclusion criteria for sepsis and whose parents gave informed consent [2]. There was 34 positive blood with higher mortality occurring in early-onset neonatal sepsis. Coagulase-negative staphylococci (32.4\%) were the predominant organism, followed by $S$. aureus $(26.5 \%)$ and $K$. pneumoniae (23.5\%). In vitro antibiotic efficacy 
was displayed with meropenem, ciprofloxacin, and clindamycin amongst others. Low antimicrobial usage and the recent existence of the hospital may be factors attributing to the decreased rates of resistance from this study.

A microbiologic surveillance study of clinical neonatal admissions from 1998-2013 at Kilifi County Hospital, Kenya, assessed the association of CoNS with prolonged admission and fatality rate [6]. Out of 9,552 neonates, about 2,271 were positive cultures with CoNS accounting for $43.8 \%$ of the isolates and $15.1 \%$ mortality. Of all the 995 CoNS isolates, $98.8 \%$ were sampled within the first 48 hours of admission. Based on the high rate of CoNS isolation, the study suggests that they were contaminants during blood sample collection.

\section{Discussion}

Neonatal sepsis is often classified as early or late onset based on the age of the child [1]. With the high morbidity and mortality associated with neonatal sepsis, empirical treatment is essential and requires indepth knowledge of the bacterial spectrum and antibiotic susceptibility pattern prevalent in the hospital location $[2,3,10]$. However, despite the initiation of empirical treatment, neonatal sepsis still remains a vital cause of mortality and morbidity globally because of the non-specific clinical manifestations [4]. It is becoming marked with high drugresistant pathogens due to the rampant use of antibiotics and wrong therapeutic regimens. Biomarkers such as procalcitonin and C-reactive protein also aid in clinical diagnosis [4,9]. Controlled use of antibiotics, restricted application of medical invasive devices, minimal handling of neonates and thorough hand hygiene can curb the mortality rate.

Hospitalization in neonates due to nosocomial CoNS bloodstream infection is common among very low birth weight infants and results in long hospital stay $[4,8]$. The range of organisms isolated from neonatal sepsis varies based on country, time of onset of illness and indigenous use of antibiotics. However, the isolation rate from the neonates was high with Coagulase-negative staphylococci and Klebsiella spp. being the predominant bacteria from most of the studies [1,3,5]. Staphylococcus species are frequently observed opportunistic pathogens in early-onset neonatal sepsis [2].

Coagulase-negative staphylococci are often considered to be contaminants except in the presence of true clinical infection with or without two positive blood cultures yielding same CoNS species $[3,9]$. According to Tomar, et al. [9], CoNS were considered as contaminants since the clinical course was not consistent with sepsis; and the simultaneous collection of two blood cultures was useful in ruling out contamination and the detection of neonatal sepsis. The role of CoNS in sepsis still remains difficult to ascertain as CoNS blood culture positivity varies in the different studies. It is often attributed to the prolonged use of medical devices, biofilm formation and increased antibiotic resistance $[2,10]$. Observing the clinical picture and other markers of infection can aid diagnosis. With the rise in CoNS isolation, the collection of two simultaneous blood cultures can aid in ruling out possible contamination [9].

According to Seale et al., the probability of isolating CoNS from blood culture decreased with the increasing volume of blood [6]. Isolation of CoNS was also closely associated with neonatal convulsions and this may be attributed to skin contamination and more difficulty in blood collection due to muscular movement during the convulsion. In late-onset neonatal sepsis, CoNS is the major pathogen especially in infants born at a lower gestational age [4]. There is a rising trend of $S$. epidermidis in neonatal sepsis when compared to other CoNS and Gram-positive cocci [1,8]. Multidrug-resistant clone of $S$. capitis has been reported in neonatal intensive care units and the NRCS-A clone was characterized using pulsed-field gel electrophoresis and MALDI-TOF MS in Belgium, Australia, United Kingdom, Sweden, and France. This also showed increased resistance to frequently used penicillin, cephalosporins, vancomycin and aminoglycosides in neonates $[7,8]$. In resource-poor settings, CoNS may emerge as an opportunistic pathogen with the increased use of invasive techniques during neonatal care [6].

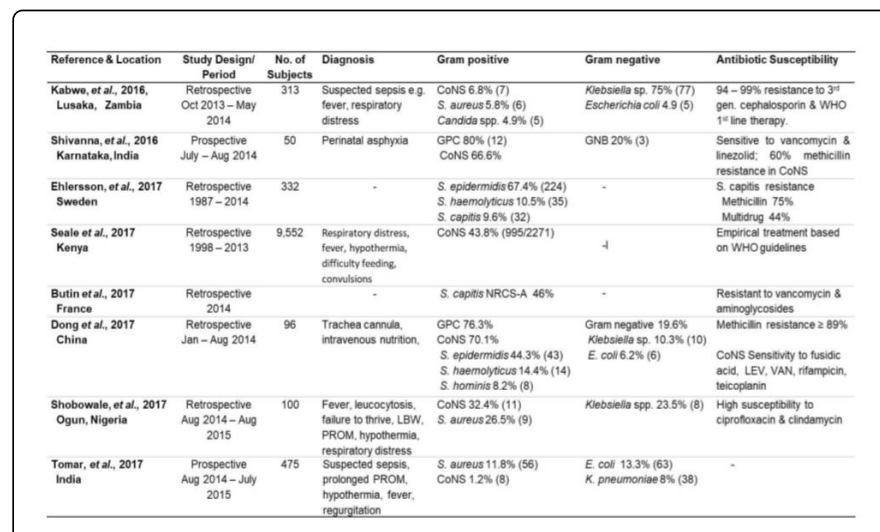

Figure 2: A review of bacterial isolates and antimicrobial susceptibility in neonatal sepsis. GPC: Gram-positive cocci, GNB: Gram-negative bacilli, CoNS: Coagulase-Negative Staphylococci, LBW: Low Birth Weight, RD: Respiratory Distress, PROM: Premature Rupture of Membrane, WHO: World Health Organisation, VAN: Vancomycin, LEV: Levofloxacin, '-': Not Indicated.

Antibiotic resistance exhibited among the Gram-positive cocci includes oxacillin, penicillin, ampicillin, and erythromycin; however, high sensitivity rate was observed in vancomycin, rifampicin and fusidic acid. S. haemolyticus had $100 \%$ resistance to penicillin, ampicillin, and erythromycin while it varied in $S$. epidermidis except for ampicillin [1]. According to Shivanna, et al., $60 \%$ of the CoNS were methicillin resistant while they were all sensitive to linezolid and vancomycin. Vancomycin was the most effective antibiotic to all the CoNS and Gram-positive cocci $[1,8]$.

\section{Conclusion}

Gram-positive cocci were the main causative pathogens in neonatal sepsis with CoNS being predominant. Coagulase-negative staphylococci such as $S$. epidermidis, S. haemolyticus, and S. capitis are opportunistic pathogens and the leading bacterial isolates with low susceptibility often observed to commonly used antibiotics. Multiple antibiotic resistance in nosocomial infection is closely associated with neonatal sepsis and increased mortality. There is a need for general use of automated blood culture systems and surveillance at regular intervals, as a countermeasure for the emergence and spread of multiple antibiotic resistance.

\section{Future Direction}

In summary, Coagulase-negative staphylococci in neonatal sepsis will be a thriving area of research for years to come. With the rate of colonization of CoNS still on the rise in neonatal sepsis and its close 
Citation: Grace JA, Obaro SK (2019) A Systematic Review of Coagulase-Negative Staphylococci in Neonatal Sepsis. Clin Microbiol 8: 326. doi:

Page 4 of 4

association with hospital routine, there is a need to address the problem by implementing strict surveillance and infection control measures. Initiating a policy on the collection of two simultaneous blood cultures can aid in ruling out possible contamination with CoNS. Laboratory investigation on the global spread of the multidrugresistant NRCS-A clone of $S$. capitis is required to unravel the route of dissemination and its reservoir, in a bid to minimize spread. Alternate antimicrobial treatment plan needs to be investigated for neonatal sepsis associated with NRCS-A clone. Introduction of vaccination opportunities can aid in the management of CoNS as pathogens. Adequate funding and collaboration of research effort will be required to address these key questions.

\section{References}

1. Dong H, Cao H, Zheng H (2017) Pathogenic bacteria distributions and drug resistance analysis in 96 cases of neonatal sepsis. BMC Pediatrics $17: 44$.

2. Shobowale EO, Solarin AU, Elikwu CJ, Onyedibe KI, Akinola IJ, et al. (2017) Neonatal sepsis in a Nigerian private tertiary hospital: Bacterial isolates, risk factors and antibiotic susceptibility patterns. Ann Afr Med 16: 52-58.

3. Shivanna V, Sunkappa SR, Venkatesha D (2016) The rising trend of coagulase-negative staphylococci in neonatal septicemia. Ind J Path Microbiol 59: 510-512.
4. Marchant EA, Boyce GK, Sadargani M, Lavoie PM (2013) Neonatal sepsis due to coagulase-negative staphylococci. Clin Develop Immun 2013: 586076.

5. Kabwe M, Tembo J, Chilukutu L, Chilufya M, Ngulube F, et al. (2016) Etiology, antibiotics resistance and risk factors for neonatal sepsis in a large referral center in Zambia. Pediatric Infect Dis 35: e191-198.

6. Seale AC, Obiero CW, Jones KD, Barsosio HC, Thitiri J, et al. (2017) Should first-line empiric treatment strategies for neonates cover coagulase-negative staphylococcal infections in Kenya? Pediatric Infect Dis J 36: 1073-1078.

7. Butin M, Martins SP, Rasigade JR, Picaud JC, Laurent F (2017) Worldwide endemicity of a multidrug-resistant Staphylococcus capitis clone involved in neonatal sepsis. Emerg Infect Dis 23: 538-539.

8. Ehlersson G, Hellmark B, Svartstrom O, Stenmark B, Soderquist B (2017) Phenotypic characterization of coagulase-negative staphylococci isolated from blood cultures in newborn infants with a special focus on Staphylococcus capitis. Acta Paediatrica 106: 10.

9. Tomar P, Garg A, Gupta R, Singh A, Gupta NK, et al. (2017) Simultaneous two-site blood culture for diagnosis of neonatal sepsis. Ind Pediatrics 54: 199-203.

10. Heilmann C, Ziebuhr W, Becker K (2018) Are coagulase-negative staphylococci virulent? Clin Microbiol Infect. 